\title{
Two cases of venous sinus thrombosis in women taking the oral contraceptive pill, Yaz (dropirenonel ethinyl estradiol), and review of the literature
}

\author{
Margaret A. Riordan, Eric M. Deshaies* \\ Department of Neurosurgery, State University of New York Upstate Medical University, Oswego, USA; \\ *Corresponding Author: DeshaieE@upstate.edu
}

Received 29 April 2013; revised 2 June 2013; accepted 30 June 2013

Copyright (C) 2013 Margaret A. Riordan, Eric M. Deshaies. This is an open access article distributed under the Creative Commons Attribution License, which permits unrestricted use, distribution, and reproduction in any medium, provided the original work is properly cited.

\begin{abstract}
Cerebral venous sinus thrombosis (CVT) is a rare cause of stroke and the clinical presentation is variable, thus mimicking other neurologic pathologies. One of the more common acquired risk factors of venous sinus thrombosis is oral contraceptive medications such as Yaz. While the prognosis for CVT is favorable, it is important to identify and treat early to prevent neurologic deficits. We report two cases of young female patients who presented with sinus thrombosis and review the current literature on this topic. CVT accounts for $0.5 \%$ of strokes and most commonly presents with headaches and in many cases focal neurologic deficits. Diagnosis is confirmed with neuroimaging including CT angiography or MR angiography. Treatment consists of anticoagulation and in some cases, thrombolytic therapy is beneficial. The overall prognosis of CVT is favorable but, further research is needed to outline optimal treatment options including the duration of anticoagulation therapy and the role of thrombolytic therapy to prevent serious neurologic deficits.
\end{abstract}

Keywords: Cerebral Venous Sinus Thrombosis; Oral Contraceptives; Stroke; Thrombolytic Therapy

\section{INTRODUCTION}

Cerebral venous sinus thrombosis (CVT) is a rare cause of cerebral injury, accounting for approximately $0.5 \%$ of all strokes [1-6]. In the adult population, the incidence of CVT is estimated to be around 3 - 4 cases per one million people and most commonly occurs between the ages of 20 - 40 years with a mean age of 38.7 years $[4,5]$. Presenting symptoms can be variable and may lead to misdiagnosis or delay in treatment; therefore, it is important for clinicians to consider CVT when evaluating patients, especially those at increased risk of thrombosis.

CVT is three times more prevalent in women and those taking oral contraceptive pills (OCPs) are at increased risk of venous thromboembolic events [4,7]. The World Health Organization recommends avoiding using OCPs in women who have thrombogenic mutations, who smoke, are older than 35 years of age, and who have given birth 21 days prior to starting an OCP to prevent thromboembolic complications [8]. Despite these risks, $64 \%$ of women in their reproductive age use some method of contraception, most commonly OCPs $[8,9]$. Newer generation combined OCPs such as Yaz (dropirenone/ethinyl estradiol manufactured by Bayer Pharmaceuticals), contained smaller doses of estrogen derivatives and different progestins in order to minimize negative side effects such as breakthrough bleeding, nausea, fluid retention, and thrombophilia [9-11]. The risk of venous sinus thrombosis has been shown to be similar in patients taking pills containing dropirenone and levonorgestrel, another form of progesterone associated with low thrombotic risk [8,11,12]; thombotic events were more commonly deep vein thromboses and pulmonary emboli. We describe two low risk patients taking Yaz who presented with sinus thrombosis and review the current literature on this topic.

\section{CASE PRESENTATION}

\subsection{Case One}

A 22-year-old female presented with headache and 
right arm numbness that started one day prior to admission. After a few hours, she developed shortness of breath, questionable seizure activity, and was taken to an outside hospital for examination. Upon arrival to that hospital, she was intubated for airway protection given her altered mental state and a CT scan of her head showed a hypodensity in the posterior parietal lobe suggestive of infarction; thus, she was transferred to our hospital for further treatment. Her past medical history was unremarkable and her medications included Yaz OCP's. She did not smoke and had no family history of hypercoagulopathies. On clinical exam, she had no focal neurologic deficits.

Subsequent brain Magnetic Resonance Venography (MRV) showed superior sagittal sinus (SSS) thrombosis, multifocal areas of venous infarction, and cerebral edema. A cerebral angiogram confirmed occlusion of the anterior and middle segments of the SSS. A hypercoagulability workup was negative and she was started on intravenous heparin followed by oral warfarin therapy.

Her neurological exam improved and she was discharged with slight decreased sensation in her right upper extremity. At a three month follow up, her paresthesias had resolved and an MRV showed recanalization of the SSS.

\subsection{Case Two}

A 20-year-old female presented to an outside hospital with a four day history of progressively worsening headaches. On the day of presentation, her headache was associated with nausea, vomiting, neck stiffness, and photophobia without any neurologic deficit. She denied any history of trauma or recent infection and had no significant past medical history. This patient was also taking Yaz for about one year, did not smoke, and had no other risk factors for thromboembolic events. Prior to transport to our hospital, a brain CT scan showed a hyperdensity in the right transverse sinus (TS), right cerebellar intraparenchymal hemorrhage, and subarachnoid hemorrhage.

MRV showed thrombus in the right internal jugular vein (IJV), right TS, right sigmoid sinus (SS), and right occipital hemorrhage with associated cerebral edema. The extent of thrombosis was confirmed with cerebral angiography. She was started on a heparin drip and bridged to warfarin therapy. Follow-up imaging showed mildly increased subarachnoid hemorrhage, but clinically she remained stable. Additionally, a subsequent MRI showed a venous infarct in the right temporal lobe.

Hypercoagulable workup was normal and she was discharged in good condition on warfarin. Follow-up imaging showed residual thrombus in the right sigmoid sinus, but the patient remained in stable condition with only mild intermittent headaches.

\section{DISCUSSION AND LITERATURE REVIEW}

\subsection{Signs and Symptoms}

Patients with cerebral venous sinus thrombosis can present with varying signs and symptoms that can mimic other pathologies making the diagnosis more difficult. The largest study of cerebral venous sinus thrombosis, the International Study on Cerebral Vein and Dural Thrombosis (ISCVT), was a prospective multicenter observational study of patients with CVT that included 624 adults with CVT to examine the natural history and outcome of CVT. The most common presenting symptoms were headache, paresis, papilledema, and seizure. This study found that most patients present within 4 days of symptom onset; $37.2 \%$ with an acute presentation, 55.5\% with a subacute presentation, and $7.2 \%$ with a chronic presentation. Delay in presentation can be attributed to the often vague presenting signs and symptoms and delay in diagnosis after seeking medical attention. 83.3\% of patients were acutely treated with intravenous heparin or low molecular weight heparin and at 6 months $79.6 \%$ of patients continued to take oral anticoagulation. The thirty day mortality rate was $3.4 \%$ while $79 \%$ of patients experienced complete recovery within six months [1].

Another study included 109 patients in the Middle East and outlined the natural history of CVT and showed similar results to the ISCVT study. $81 \%$ presented with headaches, $45 \%$ with focal neurologic deficits, 39\% seizures, and 37\% experienced mental status changes. $67 \%$ of these patients were treated with anticoagulation, 85\% were independent at follow up and they report an 8\% mortality rate [2].

There are several case studies in the literature that also describe the frequency of typical signs and symptoms associated with venous sinus thrombosis. A series of 41 patients diagnosed with venous sinus thrombosis was described and the most frequent presenting symptoms were headache (80\%), focal motor deficit (39\%), visual disorders (29.2\%), diplopia (21\%), coma (19.5\%), hemiparesis (12.2\%), and papilledema (9.75\%) [13]. Another series that included 38 patients showed similar results with $75 \%$ of patients presenting with headaches, $45 \%$ papilledema, 34\% hemiplegia, and 29\% had Jacksonian or Grand Mal seizures [14]. Other smaller case series present similar results as well [3,15-22]. These signs and symptoms are not unique to CVT and could explain the delay in diagnosis observed in the ISCVT trial.

\subsection{Risk Factors}

The ISCVT trial found that $43.6 \%$ of patients with CVT had at least one inherited or acquired prothrombotic conditions leading to venous sinus thrombosis $[1,23]$. Oral contraceptive (OCP) use is an acquired risk factor 
that can contribute to CVT formation and is generally associated with lower morbidity and mortality rates than other patients with CVT not taking OCPs [4,7]. Both patients described by us above were taking oral contraceptive pills, which have been known to increase a patient's risk for venous thromboembolic events including CVT. The odds ratio associated with OCP use is 5.59 (95\% CI 3.95 - 7.91) and CVT occurs more commonly in patients taking third generation OCPs $[7,24,25]$. The mechanism by which hormonal stimulation increases the risk of venous thrombosis is unknown. Hormone therapy does impair protein-S activity and increases prothrombin levels in the blood leading to a hypercoagulable state. OCPs may also increase levels of fibrinogen and factors VII, VIII, and X thereby increasing a patient's risk of venous thrombosis $[7,24]$.

Peurperium states are also associated with increased risk of CVT. Pregnancy induces a prothrombotic state that can be exacerbated by delivery trauma and dehydration associated with labor. CVT is more common in the third trimester and for six to eight weeks postpartum. Approximately $2 \%$ of pregnancy-associated strokes are due to CVT [23].

Other acquired risk factors include CNS malignancy and CNS infection; both are associated with a worse prognosis [7]. In the ISCVT trial, 7.4\% of patients were also diagnosed with malignancy and $12.3 \%$ with infections; the greatest frequency of infections was in the head and neck regions [1].

Inherited risk factors can also increase risk of developing CVT. Factor-V Leiden and activated protein-C resistance are the most prevalent hereditary mutations associated with CVT $[7,26]$. Prevalence of these mutations range from $10 \%-12.4 \%$ of cases. Prothrombin G20210A mutation is found in $2 \%-3 \%$ of healthy individuals and causes higher than normal levels of circulating prothrombin leading to increased incidence of venous thrombotic events. In case control studies it has been found to be associated with CVT in $11 \%$ - $20 \%$ of patients $[7,23]$. Other less common hereditary mutations found in patients with CVT include hyperhomocysteinemia, protein-C, protein-S, and antithrombin deficiencies [7,23].

\subsection{Pathogenesis}

There are two pathophysiologic mechanisms proposed that cause the signs and symptoms described in CVT: venous occlusion with infarction and intracranial hypertension secondary to resulting hydrocephalus. Occlusion of cerebral veins causes localized cerebral edema and venous infarction leading to ischemic neuronal damage and petechial hemorrhages which can merge to become large hematomas. Additionally, venous congestion causes a rise in intracranial pressure, a decrease in capillary perfusion pressure, and increase in blood volume. These changes lead to high venous system pressure and disruption of the blood brain barrier causing vasogenic edema. Cytotoxic edema also develops due to failure of the $\mathrm{Na}^{+} / \mathrm{K}^{+}$ATPase dependent pump. Furthermore, inflammatory cell infiltration at the site of thrombosis can exacerbate cerebral edema [27].

Secondly, occlusion of the major venous drainage pathway leads to the development of intracranial hypertension by blocking the normal cerebrospinal fluid drainage pathways. Obstruction of the cerebral sinuses inhibits CSF drainage from the arachnoid granulations leading to elevated ICP and worsening brain injury [5,27-29].

\subsection{Radiographic Findings}

CVT can be difficult to diagnose given the vague symptomatology and the large spectrum of clinical presentations; thus, imaging studies can aid in the diagnosis and exclude other benign etiologies. Non-enhanced CT scans can quickly rule out other causes of the patient's presenting symptoms and are commonly the first diagnostic test done in the emergency room. Classically, CT scans of patients with sinus thrombosis depict a hyperatenuating thrombus in the occluded sinuses; however, this sign is only present in approximately $25 \%$ of patients $[4,17$, 29]. Hyperattenuated sinuses can also be found in patients with dehydration, elevated hematocrit, or adjacent hemorrhage.

Contrast enhanced CT scans can provide additional information. Filling defects in the sinus also known as the "empty delta sign" suggest thrombosis; however, this sign is only present in $29 \%$ of patients [4,5,22,29]. While CT scans are quick tests to obtain in an emergency room setting to assess the extent of parenchymal injury, CT and MR angiography are helpful to demonstrate the extent of thrombosis.

CT or MR angiography allow for direct visualization of the cerebral vasculature and can be used as a confirmatory test. While MR venography has been considered a gold standard, CT angiography takes less than one minute to obtain images and is less affected by motion artifact. In a direct comparison of the two imaging modalities in 30 patients with CVT, thrombus was detected in the same patients; however, CT angiography detected more extensive thrombus in these patients. The correlation coefficient between the two imaging modalities was 0.7913. When using MR angiography as the gold standard, CT angiography had a sensitivity of $91.4 \%$ and a specificity of $93.7 \%$ [17]. Conventional catheter-based angiography remains the gold-standard for imaging the arterial and venous phases and also for assessing flow and hemodynamic alterations.

MR imaging can be useful to determine the age of the thrombus. Acute thrombus is isointense on T1 weighted imaging and hypointense on $\mathrm{T} 2$ due to the presence of 
deoxyhemoglobin in the thrombus. $10 \%-30 \%$ of patients present with acute thrombus on MR imaging. Subacute thrombus is hyperintense on $\mathrm{T} 1$ and $\mathrm{T} 2$ weighted images due to the presence of methemoglobin and is detected in $55 \%$ of patients at time of presentation. Chronic thrombus has variable imaging presentations and may or may not show evidence of recanalization. GRE sequences can also detect the presence of blood breakdown products and can be useful in diagnosing CVT [29].

The most common sinuses affected are the superior sagittal sinus, transverse, sigmoid, and deep venous system. Multiple thrombi are present in up to $90 \%$ of patients [1,4,23,28-31].

Parenchymal injury in association with CVT is present in approximately $35 \%-57 \%$ of patients $[16,17,29]$. Focal edema can be detected on CT and MR imaging in approximately $8 \%$ and $25 \%$ of cases respectively [29]. One study found a correlation between severe cognitive deficits and thalamic injury seen on imaging studies [16]. Areas of hemorrhage occur in $30 \%$ - $60 \%$ of patients with CVT. Hemorrhage in the frontal or parietal lobes suggests superior sagittal sinus thrombosis whereas hemorrhage in the temporal or occipital lobes is more common with transverse sinus thrombosis $[17,29]$.

\subsection{Other Diagnostic Tests}

Measuring d-dimer, a product of fibrin degradation, has been used to exclude diagnosis of DVT and pulmonary embolus; however, the specificity is poor given the multiple causes for elevated d-dimer. As CVT occurs due to similar hemodynamic abnormalities, d-dimer measurements have been studied as a screening tool. A multicenter prospective study examined d-dimer sensitivity in the evaluation of CVT in which 35 patients were diagnosed with CVT and 34 had a positive d-dimer. When comparing to 308 patients without CVT, 27 had positive d-dimers, yielding a sensitivity of $97.1 \%$, specificity of $91.2 \%$, negative predictive value of $99.6 \%$, and positive predictive value of $55.7 \%$ [32]. Negative d-dimer levels can help rule out CVT in patients with low suspicion for the diagnosis [23].

\subsection{Medical Management}

Heparin was first used as a treatment for CVT in 1941, but this therapy remained controversial for many years due to the possibility of spontaneous cerebral hemorrhage despite the risk of increasing thrombus size without anticoagulation treatment. The first randomized control trial of unfractionated heparin versus placebo was stopped after 20 patients were enrolled as there was a clear benefit seen in patients receiving heparin treatment. Eight patients receiving heparin treatment versus two in the placebo group had a good outcome; furthermore, three patients in the placebo group died demonstrating a clinical benefit to heparin therapy over placebo [33].

A follow-up randomized placebo control study by de Bruijn et al. [34] of low molecular weight heparin in 60 patients found no significant difference in outcome between the placebo and treatment group. A poor outcome was seen in $20 \%$ of the treated group and $24 \%$ of the placebo group; however, the treatment group trended towards improved outcome after 12 weeks of treatment. Additionally, there were no increases in cerebral hemorrhage in either study [33,35]. While there was no significant difference between the two treatment groups, anticoagulation treatment did not worsen outcome and may be used effectively to treat CVT [23].

To compare the efficacy of fractionated versus unfractionated heparin, a prospective cohort study using data from the ISCVT suggested better efficacy and safety profile when comparing the two forms of heparin [20]. Based on these and other smaller case series, patients with CVT can be treated effectively with both forms of heap$\operatorname{rin}[34,36,37]$.

Oral anticoagulation should be initiated following acute treatment with heparin. If the patient does not have inherited thrombophilia or recurrent thrombosis, three to six months of therapy may be sufficient [21,34]; however, there is no class one evidence supporting any particular length of treatment.

Anti-epileptic drugs are recommended in the acute phase of injury by the AHA and ASA if a patient suffers one episode of epileptic activity [23]. Based on the ISCVT study, 39.3\% of patients with CVT developed seizure activity; focal (9.3\%), generalized (19.7\%), both $(10.3 \%)$, or status epilepticus $(0.5 \%)$. Seizure activity was more common in patients with motor deficits, cerebral lesion such as a hemorrhage, or superior sagittal sinus or cortical vein thrombosis [38].

\subsection{Thrombolytics}

Although most patients suffering from CVT are successfully managed with systemic anticoagulants, some patients worsen, requiring further treatment with local thrombolytics or mechanical thrombectomy. Wasay et al. [39] compared local urokinase administration to systemic heparin in 40 patients. 20 patients were treated with endovascular administration of urokinase via the femoral vein into the superior sagittal sinus followed by treatment with systemic heparin. These patients were compared to 20 patients treated with systemic heparin alone and no worsening neurologic deficit or worsening hemorrhage was noted; in fact, neurologic exam at discharge was better than patients who were treated with heparin only.

Similarly, a prospective study of 20 patients with CVT examined outcomes after endovascular treatment with 
urokinase and mechanical thrombectomy (15 patients) followed by systemic heparin or LMWH therapy. These patients were selected because they were assumed to have a poor prognosis if treated with systemic anticoagulation alone based on altered mental status, coma, straight sinus thrombosis, or large space occupying lesions. Despite the initial poor prognosis, 12 patients were catagorized as having an execellent recovery or having minor handicaps. The mortality rate in this study was higher than previously published, but could be attributed to the sicker patient population [40].

A systematic review of this topic included 169 patients and analyzed the type of thrombolytic used, method of delivery and outcome. $88 \%$ of these patients were treated with local administration versus system administration of the thrombolytic and 56\% received either a single dose or infusion lasting $<24 \mathrm{hrs}$. Urokinase was used in $75 \%$, $22 \%$ received rtTPA, the rest received either streptokinase or multiple agents. $86 \%$ of these patients were independent at discharge which is comparable to outcomes in large case studies of all patients treated for CVT. Complications from thrombolytic treatment included extracranial hemorrhage, intracranial hemorrhage. Recanalization occurred in most patients and was less likely to occur in those patients treated with systemic thrombolytics [39,41-44]. Based on these studies, thrombolytics may benefit some patients for which anticoagulation therapy does not prove sufficient treatment.

Direct thrombectomy can be considered in patients who do not respond to medical management. Several case repots in the literature describe successful mechanical thrombolysis of the thrombus followed by direct thrombolytic administration [45-51]. For example, case reports using the AngioJet device, a water suctioning device commonly used in cardiac catheterization, has been shown to successfully disrupt cerebral venous sinus thrombosis without worsening clinical outcome [45,52]. Despite initial success, these cases represent a publication bias and further research is needed before such interventions become standard of care.

\subsection{Long Term Evaluation and Prognosis}

Approximately $80 \%$ of patients with CVT have a good outcome. Mortality rates reported in the literature range from $2 \%-8 \%$ and $65 \%$ - $90 \%$ of patients make a complete recovery with minimal neurologic deficit $[1-3,13$, 14,16,22,34,53,54]. In multiple studies, poor prognosis correlated with male gender, older age, coma, malignnancy, CNS infection, and worsening exam after admission [44,53]. Pregnancy, peurperium, and oral contraceptive use are associated with a better outcome than those patients with systemic disease such as malignancy or inherited thrombophilia $[4,16]$.

The recurrence rate of venous thromboembolic events ranges from $5 \%-13 \%$ and such events usually recurred within one year of the original CVT. Long term follow up based on the ISCVT study found that $5.8 \%$ of patients had a recurrent venous thromboembolic event and that $63.2 \%$ occurred in the first year. Of these patients, $2.2 \%$ had recurrence CVT, while the rest had either deep venous thrombosis or pulmonary embolism. Risk factors for recurrence include male gender and polycythemia or thrombocythemia [55].

Similar results were found in other smaller retrospective studies. Martinelli et al. [31] found a recurrence rate of $10 \%$ and of these $3 \%$ had recurrent CVT and recurrence was higher in males. Gosk-Bierska et al. [54] found $13 \%$ recurrence of venous thromboembolism with a median time to recurrence of 10 months. Ferro et al. [53] found a recurrence rate of $2 \%$.

\section{CONCLUSION}

Cerebral venous sinus thrombosis is a rare cause of stroke that presents with variable symptoms that can mimic other neurologic pathologies. Important risk factors include genetic prothrombotic states, peurperium states, use of oral contraceptive medications, and infection. Prompt verification of the thrombosis with neuroimaging such as MR or CT angiography is necessary to provide adequate treatment in a timely manner. Further research is needed to define the duration of anticoagulation therapy as well as the role for endovascular thrombolysis.

\section{REFERENCES}

[1] Ferro, J.M., Canhão, P., Stam, J., Bousser, M.-G. and Barinagarrementeria, F. (2004) Prognosis of cerebral vein and dural sinus thrombosis: Results of the International Study on Cerebral Vein and Dural Sinus Thrombosis (ISCVT). Stroke: A Journal of Cerebral Circulation, 35, 664-670. doi:10.1161/01.STR.0000117571.76197.26

[2] Khealani, B.A., Wasay, M., Saadah, M., Sultana, E., Mustafa, S., Khan, F.S. and Kamal, A.K. (2008) Cerebral venous thrombosis: A descriptive multicenter study of patients in Pakistan and Middle East. Stroke: A Journal of Cerebral Circulation, 39, 2707-2711. doi:10.1161/STROKEAHA.107.512814

[3] Christo, P.P., Carvalho, G.M. de and Gomes Neto, A.P. (2010) Cerebral venous thrombosis: Study of fifteen cases and review of literature. Revista da Associação Médica Brasileira (1992), 56, 288-292. doi:10.1590/S0104-42302010000300011

[4] Ehtisham, A. and Stern, B.J. (2006) Cerebral venous thrombosis: A review. The neurologist, 12, 32-38. doi:10.1097/01.nrl.0000178755.90391.b6

[5] Filippidis, A., Kapsalaki, E., Patramani, G. and Fountas, K.N. (2009) Cerebral venous sinus thrombosis: Review of the demographics, pathophysiology, current diagnosis, and treatment. Neurosurgical Focus, 27, E3. doi:10.3171/2009.8.FOCUS09167 
[6] Bentley, J.N., Figueroa, R.E. and Vender, J.R. (2009) From presentation to follow-up: Diagnosis and treatment of cerebral venous thrombosis. Neurosurgical Focus, 27, E4. doi:10.3171/2009.8.FOCUS09166

[7] McBane, R.D., Tafur, A. and Wysokinski, W.E. (2010) Acquired and congenital risk factors associated with cerebral venous sinus thrombosis. Thrombosis Research, 126, 8187. doi:10.1016/j.thromres.2010.04.015

[8] Sehovic, N. and Smith, K.P. (2010) Risk of venous thromboembolism with drospirenone in combined oral contraceptive products. The Annals of Pharmacotherapy, 44, 898-903. doi:10.1345/aph.1M649

[9] Bachmann, G. and Kopacz, S. (2009) Drospirenone/ethinyl estradiol 3 mg/20 mug (24/4 day regimen): Hormonal contraceptive choices - Use of a fourth-generation progestin. Patient Preference and Adherence, 3, 259-264. doi:10.2147/PPA.S3901

[10] Kaunitz, A.M., Burkman, R.T., Fisher, A.C. and Laguardia, K.D. (2009) Cycle control with a 21-day compared with a 24-day oral contraceptive pill: A randomized controlled trial. Obstetrics and Gynecology, 114, 1205-1212. doi:10.1097/AOG.0b013e3181beab47

[11] Rapkin, A.J., Sorger, S.N. and Winer, S.A. (2008) Drospirenone/ethinyl estradiol. Drugs of Today (Barcelona, Spain: 1998), 44, 133-145. doi:10.1358/dot.2008.44.2.1191057

[12] López, M., Vayá, A., Martínez Triguero, M.L., Contreras, M.T., Todolí, J., Ricart, A. and Laiz, B. (2009) Yasmin and venous thromboembolism: New case reports. Clinical Hemorheology and Microcirculation, 42, 65-69.

[13] Sahraian, M.A., Akbari, H., Khajavi, M.R., Najafi, A. and Khashayar, P. (2010) The risk factors and the treatment course of cerebral venous thrombosis: An experience of 41 cases. Acta Neurologica Belgica, 110, 230-233.

[14] Bousser, M.G., Chiras, J., Bories, J. and Castaigne, P. (1985) Cerebral venous thrombosis-A review of 38 cases. Stroke: A Journal of Cerebral Circulation, 16, 199213. doi:10.1161/01.STR.16.2.199

[15] Fischer, C., Goldstein, J. and Edlow, J. (2010) Cerebral venous sinus thrombosis in the emergency department: Retrospective analysis of 17 cases and review of the literature. The Journal of Emergency Medicine, 38, 140-147. doi:10.1016/j.jemermed.2009.08.061

[16] Appenzeller, S., Zeller, C.B., Annichino-Bizzachi, J.M., Costallat, L.T.L., Deus-Silva, L., Voetsch, B. and Cendes, F. (2005) Cerebral venous thrombosis: Influence of risk factors and imaging findings on prognosis. Clinical Neurology and Neurosurgery, 107, 371-378. doi:10.1016/j.clineuro.2004.10.004

[17] Khandelwal, N., Agarwal, A., Kochhar, R., Bapuraj, J.R., Singh, P., Prabhakar, S. and Suri, S. (2006) Comparison of CT venography with MR venography in cerebral sinovenous thrombosis. AJR. American Journal of Roentgenology, 187, 1637-1643. doi:10.2214/AJR.05.1249

[18] Shahsavarzadeh, T., Javanmard, S.H. and Saadatnia, M. (2011) Impact of factor VIII and von Willebrand factor plasma levels on cerebral venous and sinus thrombosis: Are they independent risk factors? European Neurology, 66, 243-246. doi:10.1159/000331048
[19] Soleau, S.W., Schmidt, R., Stevens, S., Osborn, A. and MacDonald, J.D. (2003) Extensive experience with dural sinus thrombosis. Neurosurgery, 52, 534-544. doi:10.1227/01.NEU.0000047815.21786.C1

[20] Coutinho, J.M., Ferro, J.M., Canhão, P., Barinagarrementeria, F., Bousser, M.-G. and Stam, J. (2010) Unfractionated or low-molecular weight heparin for the treatment of cerebral venous thrombosis. Stroke: A Journal of Cerebral Circulation, 41, 2575-2580. doi:10.1161/STROKEAHA.110.588822

[21] Galarza, M. and Gazzeri, R. (2009) Cerebral venous sinus thrombosis associated with oral contraceptives: The case for neurosurgery. Neurosurgical Focus, 27, E5. doi:10.3171/2009.8.FOCUS09158

[22] Bousser, M.G. (2000) Cerebral venous thrombosis: Diagnosis and management. Journal of Neurology, 247, 252258. doi:10.1007/s004150050579

[23] Saposnik, G., Barinagarrementeria, F., Brown Jr., R.D., Bushnell, C.D., Cucchiara, B., Cushman, M. and Tsai, F.Y. (2011) Diagnosis and management of cerebral venous thrombosis: A statement for healthcare professionals from the American Heart Association/American Stroke Association. Stroke: A Journal of Cerebral Circulation, 42, 1158-1192. doi:10.1161/STR.0b013e31820a8364

[24] Dunne, C., Malyuk, D. and Firoz, T. (2010) Cerebral venous sinus thrombosis in a woman using the etonogestrelethinyl estradiol vaginal contraceptive ring: A case report. Journal of Obstetrics and Gynaecology Canada: JOGC = Journal D'obstétrique et Gynécologie du Canada: JOGC, 32, 270-273.

[25] Kolacki, C. and Rocco, V. (2012) The combined vaginal contraceptive ring, nuvaring, and cerebral venous sinus thrombosis: A case report and review of the literature. The Journal of Emergency Medicine, 42, 413-416. doi:10.1016/j.jemermed.2011.06.011

[26] Ben Salem-Berrabah, O., Fekih-Mrissa, N., N’siri, B., Ben Hamida, A., Benammar-Elgaaied, A., Gritli, N. and Mrissa, R. (2012) Thrombophilic polymorphisms-factor V Leiden G1691A, prothrombin G20210A and MTHFR C677Tin Tunisian patients with cerebral venous thrombosis. Journal of Clinical Neuroscience: Official Journal of the Neurosurgical Society of Australasia, 19, 1326-1327. doi:10.1016/j.jocn.2011.11.029

[27] Nagai, M., Terao, S., Yilmaz, G., Yilmaz, C.E., Esmon, C. T., Watanabe, E. and Granger, D.N. (2010) Roles of inflammation and the activated protein $\mathrm{C}$ pathway in the brain edema associated with cerebral venous sinus thrombosis. Stroke: A Journal of Cerebral Circulation, 41, 147152. doi:10.1161/STROKEAHA.109.562983

[28] Stam, J. (2005) Thrombosis of the cerebral veins and sinuses. The New England Journal of Medicine, 352, 17911798. doi:10.1056/NEJMra042354

[29] Leach, J.L., Fortuna, R.B., Jones, B.V. and Gaskill-Shipley, M.F. (2006) Imaging of cerebral venous thrombosis: Current techniques, spectrum of findings, and diagnostic pitfalls. Radiographics: A Review Publication of the Radiological Society of North America, Inc., 26, S19-S41. doi:10.1148/rg.26si055174

[30] Crassard, I., Soria, C., Tzourio, C., Woimant, F., Drouet, 
L., Ducros, A. and Bousser, M.-G. (2005) A negative Ddimer assay does not rule out cerebral venous thrombosis: A series of seventy-three patients. Stroke: A Journal of Cerebral Circulation, 36, 1716-1719. doi:10.1161/01.STR.0000173401.76085.98

[31] Martinelli, I., Bucciarelli, P., Passamonti, S.M., Battaglioli, T., Previtali, E. and Mannucci, P.M. (2010) Longterm evaluation of the risk of recurrence after cerebral sinus-venous thrombosis. Circulation, 121, 2740-2746. doi:10.1161/CIRCULATIONAHA.109.927046

[32] Kosinski, C.M., Mull, M., Schwarz, M., Koch, B., Biniek, R., Schläfer, J. and Schiefer, J. (2004) Do normal D-dimer levels reliably exclude cerebral sinus thrombosis? Stroke: A Journal of Cerebral Circulation, 35, 2820-2825. doi:10.1161/01.STR.0000147045.71923.18

[33] Einhäupl, K.M., Villringer, A., Meister, W., Mehraein, S., Garner, C., Pellkofer, M. and Schmiedek, P. (1991) Heparin treatment in sinus venous thrombosis. Lancet, 338, 597-600. doi:10.1016/0140-6736(91)90607-Q

[34] Coutinho, J.M. and Stam, J. (2010) How to treat cerebral venous and sinus thrombosis. Journal of Thrombosis and Haemostasis: JTH, 8, 877-883. doi:10.1111/j.1538-7836.2010.03799.x

[35] De Bruijn, S.F. and Stam, J. (1999) Randomized, placebo-controlled trial of anticoagulant treatment with low molecular-weight heparin for cerebral sinus thrombosis. Stroke: A Journal of Cerebral Circulation, 30, 484-488. doi:10.1161/01.STR.30.3.484

[36] Ferro, J.M. and Canhão, P. (2008) Acute treatment of cerebral venous and dural sinus thrombosis. Current treatment options in neurology, 10, 126-137. doi:10.1007/s11940-008-0014-0

[37] Coutinho, J., de Bruijn, S.F., Deveber, G. and Stam, J. (2011) Anticoagulation for cerebral venous sinus thrombosis. Cochrane Database of Systematic Reviews (Online), 8, CD002005. doi:10.1002/14651858.CD002005.pub2

[38] Ferro, J.M., Canhão, P., Bousser, M.-G., Stam, J. and Barinagarrementeria, F. (2008) Early seizures in cerebral vein and dural sinus thrombosis: Risk factors and role of antiepileptics. Stroke: A Journal of Cerebral Circulation, 39, 1152-1158. doi:10.1161/STROKEAHA.107.487363

[39] Wasay, M., Bakshi, R., Kojan, S., Bobustuc, G., Dubey, N. and Unwin, D.H. (2001) Nonrandomized comparison of local urokinase thrombolysis versus systemic heparin anticoagulation for superior sagittal sinus thrombosis. Stroke: A Journal of Cerebral Circulation, 32, 2310-2317. doi:10.1161/hs1001.096192

[40] Stam, J., Majoie, C.B.L.M., van Delden, O.M., van Lienden, K.P. and Reekers, J.A. (2008) Endovascular thrombectomy and thrombolysis for severe cerebral sinus thrombosis: A prospective study. Stroke: A Journal of Cerebral Circulation, 39, 1487-1490.

doi:10.1161/STROKEAHA.107.502658

[41] Canhão, P., Falcão, F. and Ferro, J.M. (2003) Thrombolytics for cerebral sinus thrombosis: A systematic review. Cerebrovascular Diseases (Basel, Switzerland), 15, 159-166. doi:10.1159/000068833

[42] Kim, S.Y. and Suh, J.H. (1997) Direct endovascular thrombolytic therapy for dural sinus thrombosis: Infusion of alteplase. AJNR. American Journal of Neuroradiology, 18, 639-645.

[43] Horowitz, M., Purdy, P., Unwin, H., Carstens 3rd, G., Greenlee, R., Hise, J. and Samson, D. (1995) Treatment of dural sinus thrombosis using selective catheterization and urokinase. Annals of Neurology, 38, 58-67. doi:10.1002/ana.410380112

[44] Medel, R., Monteith, S.J., Crowley, R.W. and Dumont, A.S. (2009) A review of therapeutic strategies for the management of cerebral venous sinus thrombosis. Neurosurgical Focus, 27, E6. doi:10.3171/2009.8.FOCUS09154

[45] Agner, C., Deshaies, E.M., Bernardini, G.L., Popp, A.J. and Boulos, A.S. (2005) Coronary angiojet catheterizetion for the management of dural venous sinus thrombosis. Technical note. Journal of neurosurgery, 103, 368371. doi:10.3171/jns.2005.103.2.0368

[46] Curtin, K.R., Shaibani, A., Resnick, S.A., Russell, E.J. and Simuni, T. (2004) Rheolytic catheter thrombectomy, balloon angioplasty, and direct recombinant tissue plasminogen activator thrombolysis of dural sinus thrombosis with preexisting hemorrhagic infarctions. AJNR. American Journal of Neuroradiology, 25, 1807-1811.

[47] Nimjee, S.M., Powers, C.J., Kolls, B.J., Smith, T., Britz, G.W. and Zomorodi, A.R. (2011) Endovascular treatment of venous sinus thrombosis: A case report and review of the literature. Journal of Neurointerventional Surgery, 3, 30-33. doi:10.1136/jnis.2010.002469

[48] Chow, K., Gobin, Y.P., Saver, J., Kidwell, C., Dong, P. and Viñuela, F. (2000) Endovascular treatment of dural sinus thrombosis with rheolytic thrombectomy and intraarterial thrombolysis. Stroke: A Journal of Cerebral Circulation, 31, 1420-1425. doi:10.1161/01.STR.31.6.1420

[49] Chaloupka, J.C., Mangla, S. and Huddle, D.C. (1999) Use of mechanical thrombolysis via microballoon percutaneous transluminal angioplasty for the treatment of acute dural sinus thrombosis: Case presentation and technical report. Neurosurgery, 45, 650-656. doi:10.1097/00006123-199909000-00045

[50] Rahman, M., Velat, G.J., Hoh, B.L. and Mocco, J. (2009) Direct thrombolysis for cerebral venous sinus thrombosis. Neurosurgical Focus, 27, E7. doi:10.3171/2009.7.FOCUS09146

[51] Chahlavi, A., Steinmetz, M.P., Masaryk, T.J. and Rasmussen, P.A. (2004) A transcranial approach for direct mechanical thrombectomy of dural sinus thrombosis. Report of two cases. Journal of Neurosurgery, 101, 347-351. doi:10.3171/jns.2004.101.2.0347

[52] Dashti, S.R., Hu, Y.C., Yao, T., Fiorella, D., Mitha, A.P., Albuquerque, F.C. and McDougall, C.G. (2011) Mechanical thrombectomy as first-line treatment for venous sinus thrombosis: Technical considerations and preliminary results using the AngioJet device. Journal of Neurointerventional Surgery, 5, 49-53. doi:10.1136/neurintsurg-2011-010112

[53] Ferro, J.M., Lopes, M.G., Rosas, M.J., Ferro, M.A. and Fontes, J. (2002) Long-term prognosis of cerebral vein and dural sinus thrombosis. Cerebrovascular Diseases 
(Basel, Switzerland), 13, 272-278. doi:10.1159/000057855

[54] Gosk-Bierska, I., Wysokinski, W., Brown Jr., R.D., Karnicki, K., Grill, D., Wiste, H. and McBane 2nd, R.D. (2006) Cerebral venous sinus thrombosis: Incidence of venous thrombosis recurrence and survival. Neurology,
67, 814-819. doi:10.1212/01.wnl.0000233887.17638.d0

[55] Miranda, B., Ferro, J.M., Canhão, P., Stam, J., Bousser, M.G., Barinagarrementeria, F. and Scoditti, U. (2010) Venous thromboembolic events after cerebral vein thrombosis. Stroke: A Journal of Cerebral Circulation, 41, 1901-1906. doi:10.1161/STROKEAHA.110.581223. 